\title{
Hilfen über Hürden
}

\section{Auch Fachhochschulabsolventen der Sozialen Arbeit können promovieren}

\author{
Rudolf Schmitt
}

Prof. Dr. Rudolf Schmitt ist

Hochschullehrer am Fachbereich Sozialwesen der Hochschule Görlitz. E-Mail r.schmitt@hs-zigr.de
Absolventen von Fachbochschulstudiengängen der Sozialen Arbeit müssen Umwege in Kauf nehmen, wenn sie den Doktortitel erwerben wollen. Doch stehen mittlerweile für das Vorhaben zumindest einige hilfreiche Informationsmöglichkeiten zur Verfügung.

Warum sollten Absolventinnen und Absolventen der Sozialen Arbeit an Fachhochschulen überhaupt promovieren? Zumindest drei Antworten können gegeben werden: Eine Promotion erweitert den biografischen Spielraum der Absolventinnen und Absolventen; die Disziplin der Sozialen Arbeit kann den Nachwuchs in der Lehre aus den eigenen Reihen der Promovierten rekrutieren; und die Profession der Sozialen Arbeit profitiert von den Forschungsanstrengungen in der Praxis und bei der Organisation der Hilfen.

Allerdings zeigt die Perspektive des Promovierens dunkle Schatten: Der Bundesbericht zur Förderung des Wissenschaftlichen Nachwuchses (BuWiN) vom März 2008 schätzt, dass an Universitäten gerade ein Drittel aller Promotionsversuche erfolgreich abgeschlossen werden - unabhängig davon, ob die Absolventinnen und Absolventen von der Universität oder der Fachhochschule kommen. Fördernde und klärende Beratung ist also nicht nur für Fachhochschul-Absolventinnen und -Absolventen wichtig. Diese Einschätzung gilt zunächst unabhängig vom eigenen Fach, denn separate Daten für die Soziale Arbeit werden nicht genannt. Überhaupt fehlen empirische Daten für typische Problemlagen des Promovierens, und so ist die die folgende Sammlung von Schwierigkeiten nur von der eigenen Erfahrung mit Promovenden in unterschiedlichen Kontexten abgeleitet:

An Fachhochschulen ist in der Regel unbekannt, dass das Anrecht von Absolventinnen und Absolventen, promovieren zu dürfen, bis auf Beschlüsse der Hochschulrektorenkonferenz von 1992 zurückreicht (Weinbach 2001) - unabhängig da- von, ob der Abschluss nun das Diplom (FH) oder der Master ist. Nur wenige Kolleginnen und Kollegen vermitteln leistungsfähigen Absolventinnen und Absolventen diese biografische Alternative.

- Das Exposé zu einem Promotionsvorhaben mit der Forschungsfrage als Weichenstellung krankt oft an der ungenügenden forschungsmethodischen Grundausbildung, wenn empirische Arbeiten im Studium nicht gefordert und theoretische Diplomarbeiten auf dem Niveau von Besinnungsaufsätzen akzeptiert werden. Sowohl zur qualitativen wie zur quantitativen Forschungsmethodik muss in der Regel (auch bei Universitäts-Absolventinnen und -Absolventen) nachgeschult werden, Grundlagentheorien müssen für die speziellen Forschungsfragen erschlossen werden. Empirisch verortete aktuelle Sammelbände der Sozialarbeitswissenschaft zeigen jedoch erfreuliche Perspektiven (Engelke et al. 2007, Gahleitner et al. 2008).

- Die Betreuung durch Erst- und Zweitgutachter ist in der Regel auch an Universitäten ungenügend und bedarf im Kontext der Sozialen Arbeit der Stärkung eines wissenschaftsinteressierten und sozialarbeitsnahen Milieus (Kolloquien, Tagungen etc.).

- Die Finanzierung einer Dissertation geschieht oft durch fortlaufende berufliche Arbeit; die Informationen über Stipendien und Graduiertenkollegien sind wenig transparent, spezifische Programme für Fachhochschul-Absolventinnen und -Absolventen selten (z. B. Mertel 1999).

- Das fehlende Promotionsrecht der Fachhochschulen verkompliziert die Situation und die Suche nach universitären Erstgutachtern (und im Fall von nicht-kooperativen Promotionsverfahren auch nach Zweitgutachtern) findet an der Schwelle zum hegemonialen universitären System mit anderen Habitus-Anforderungen 
statt. Fachhochschul-Absolventinnen und -Absolventen anderer Fächer stehen dem gleichen Problem gegenüber. Keller (2008) hat in seinem Promotionsführer für Fachhochschul-Absolventinnen und -Absolventen aller Fächer viele Hürden beschrieben - eine unbedingte Empfehlung. Allerdings entspricht kein universitärer Studiengang der an Fachhochschulen gelehrten Sozialen Arbeit, und die Orientierung wird nicht einfacher dadurch, dass Universitäten derzeit ihre Studiengänge der Sozialpädagogik in
"Soziale Arbeit « oder "Social Work « umetikettieren. Es sind jedoch in der Regel sozialpädagogisch-erziehungswissenschaftliche Ausrichtungen, die für einen wichtigen Teil von Fragestellungen der genuinen Sozialen Arbeit nicht infrage kommen.

\section{Dissertationsliste Soziale Arbeit}

Doktoranden von Fachhochschulen, die über Themen der Sozialen Arbeit eine Dissertation schreiben, versammeln sich seit einiger Zeit unter dem Dach der Deutschen Gesellschaft für Soziale Arbeit e. V., um ihre Erfahrungen auszutauschen und Probleme zu diskutieren. In der der Website der Organisation unterrichtet eine Liste über die bekanntgewordenen abgeschlossenen Dissertationen:

Nationale Mindestsicherungssysteme und europäische Integration

Von der Thematisierung der Armut und sozialen Ausgrenzung zur Offenen Methode der Koordination

Von Dr. rer. soc. Benjamin Benz

\section{Krisen-Bildung}

Aspekte des Lebenslangen Lernens in der Aus- und Weiterbildung von KriseninterventionshelferInnen

Von Dr. phil. Sebastian Roth

Handeln im Konflikt

Eine qualitativ-empirische Studie zu Kindesinteressen und professionellem Handeln in Familiengericht und Jugendhilfe Von Dr. phil. Heike Schulze

Zum Verhältnis von Sozialer Arbeit und Betreuung unter besonderer Berücksichtigung beruflicher Selbständigkeit Verberuflichungs- und Professionalisierungsprozesse am Beispiel freiberuflicher Betreuungsführung in Köln

Von Dr. phil. Anne Klüser, Dipl.Sozialarbeiterin, Dipl.Pädagogin

Soziale Arbeit und Jugendkulturen

Die Dialektik von Herrschaft und Emanzipation im Kontext des Systemischen Paradigmas der Sozialen Arbeit

Von Dr. phil. Christian Spatscheck

Gesundheitsförderung in einem Industrieunternehmen

Eine salutogenetische Perspektive

Von Dr. phil. Myriam Fröschle-Mess

\section{Langzeitarbeitslosigkeit}

Individuelle Bewältigung im gesellschaftlichen Kontext

Von Dr. Michael Mehlich

\section{Empowerment als Hilfe zur Lebensbewältigung}

Anforderungen an ein integratives Empowermentmodell für die Arbeit mit psychisch kranken Menschen in Zeiten postmoderner Gesellschaftsveränderungen

Von Dr. Dieter Röh

Die Konstruktion professioneller Identität in der Sozialen Arbeit

Theoretische Grundlagen und empirische Befunde

Von Dr. Thomas Harmsen

\section{Rechtswirkung in Organisationen}

Ergebnisse einer empirischen Feldstudie zur Wirkung des novellierten $\ 93$ BSHG auf die Erbringung personenbezogener sozialer Dienstleistungen in stationären Einrichtungen Von Dr. Matthias Nauerth

Berufsbiographien und Orientierungskerne des beruflichen Handelns in zwei unterschiedlichen Gesellschaftsformationen Veränderungen und Kontinuität des beruflichen Selbstverständnisses und Handelns von Sozialwesenprofessionellen in Ostdeutschland

Von Dr. Monika Müller

Positionierungen einer systemisch-konstruktivistischen Sozialarbeitswissenschaft

Anregungen für eine Neuorientierung in der Ausbildung Von Dr. phil. Daniela Beer

\section{Lebensqualität im Stadtquartier}

Evaluationsstudie über die Stadtteil- und Familienzentren in Offenburg

Von Dr. phil. Martin Becker

Mitbestimmung als Qualitätskriterium in Jugendfreizeiteinrichtungen?

Bedingungen bürgerschaftlicher Kompetenzbildung am Beispiel Mittelfrankens.

Von Dr. Petra Ludwig

Systemtheoretische Ansätze von Niklas Luhmann und Mario Bunge im Vergleich und deren Rezeption in der Sozialen Arbeit Von Dr. phil. Michael Klassen

Konstruktivismus - Kommunikation - Soziale Arbeit Radikalkonstruktivistische Betrachtungen zu den Bedingungen des sozialpädagogischen Interaktionsverhältnisses Von Dr. phil. Björn Kraus

Behandlung als Koproduktion

Menschen mit funktionellen Beschwerden in ambulanter ärztlicher Behandlung

Von Dr. phil. Karlheinz Ortmann

Postmoderne Sozialarbeit

Ambivalenzreflexion als ein Konzept sozialarbeitswissenschaftlicher Heuristik

Von Dr. phil. Heiko Kleve 
Diesem Beratungsbedarf stehen bereits etablierte Strukturen der Unterstützung von Promotionen in der Sozialen Arbeit gegenüber, welche die oben genannten Defizite eines nur inselartig vorhandenen wissenschaftlich interessierten Milieus und der fehlenden Informationen kompensieren: Hier müssen zunächst die seit 1998 zunächst jährlich, inzwischen halbjährlich tagenden Promotionskolloquien der Deutschen Gesellschaft für Soziale
Jahrestreffen der Deutschen Gesellschaft für Soziale Arbeit durch den Wegfall der thematischen Orientierung für alle Promovierenden geöffnet werden, eine Postersession und ein Posterpreis für Dissertationspräsentationen könnten weitere Anregungen für Promovierende stiften.

Die wichtigste Forderung derzeit ist aber an die Kolleginnen und Kollegen der Fachhochschulen zu richten: Es braucht engagierte Mitstreitende, die den Studie-

\section{»In Universitäten lauern Fallstricke, die man kennen muss «}

Arbeit e. V. unter Leitung von Prof. Dr. Albert Mühlum (Mühlum 1998) genannt werden. Im Bereich der qualitativen Forschung sind derzeit pro Jahr vier zentrale Workshops und Tagungen neben kleineren Veranstaltungen zu nennen, in denen Promovierende der Sozialen Arbeit nach einem Fachhochschulabschluss ihre Arbeiten diskutieren. Seit zwei Jahren existiert eine Mailingliste zur Promotionsförderung mit derzeit über 240 Promotionsinteressierten, Promovierenden und interessierten Kolleginnen und Kollegen aus den Fachhochschulen (Anmeldung per E-Mail: r.schmitt@hs-zigr.de). Eine Website mit Basisinformationen zur Fachhochschul-Promotion in Sozialer Arbeit steht zur Verfügung (http://www.hszigr.de/ schmitt/promotionen/index_promotion.htm), eine Broschüre ist dort herunterladbar (Schmitt 2008).

Dennoch sind Lücken der Betreuung und Beratung von Fachhochschul-Absolventinnen und -Absolventen zu konstatieren: Promovierende mit quantitativen Forschungsarbeiten sowie mit historischen und theoretischen Arbeiten finden innerhalb der Fachhochschullandschaft wenige Ansprechpartner, spezifische inhaltliche Foren (z. B. Casework, Ethik in der Sozialen Arbeit) für Promovierende der Sozialen Arbeiten existieren nicht. Daraus resultiert der Vorschlag, die verschiedenen Sektionen und Arbeitsgruppen der Deutschen Gesellschaft für Soziale Arbeit und des Fachbereichstags Soziale Arbeit als Anlaufstellen und als Möglichkeit der Präsentation für Promovierende zu nutzen. Dann könnten die renden glaubwürdig einen forschenden Habitus auch in der Lehre vorleben, die Absolventinnen und Absolventen auf diese biografische Alternative hinweisen und sich die Zeit nehmen, bei der derzeit noch unvermeidbaren Fremdplatzierung der Promovenden der Sozialen Arbeit an den Universitäten diesen die unsichtbaren Fallstricke jenes Milieus zu erklären. An jedem Fachbereich der Sozialen Arbeit sollte das Amt eines Promotionsbeauftragten eingerichtet werden, der über Möglichkeiten der Promotion regelmäßig informiert wie für die Anbahnung und Koordination von Promotionsverfahren zuständig ist. Es gibt noch viel zu tun aber mehr als ein Anfang ist gemacht.

\section{Literatur}

Bundesministerium für Bildung und Forschung (BMBF) (Hrsg.) (2008). Bundesbericht zur Förderung des Wissenschaftlichen Nachwuchses (BuWiN). Eigenverlag: Berlin.

Engelke, Ernst; Maier, Konrad; Steinert, Erika; Borrmann, Stefan; Spatscheck, Christian (Hg.) (2007). Forschung für die Praxis. Zum gegenwärtigen Stand der Sozialarbeitsforschung. Freiburg im Breisgau: Lambertus.

Gahleitner, Silke Birgitta; Gerull, Susanne; Lange, Chris; SchambachHardtke, Lydia; Petuya Ituarte, Begoña; Streblow, Claudia (Hg.) (2008). Sozialarbeitswissenschaftliche Forschung. Einblicke in aktuelle Themen. Uckerland: Schibri-Verlag.

Keller, Ansgar (2008). Promotionsführer für Fachhochschulabsolventen: Möglichkeiten und Zulassungsverfahren für eine Promotion an 70 deutschen Universitäten mit Hinweisen für Absolventen von Bachelor/Masterstudiengängen sowie Berufsakademien und zur Promotion im Ausland, 8. Aufl., Berlin, ISBN 3-931221-07-5, Berlin: Steinbeis.

Mertel, Sabine (1999) Mit der Fachhochschule aus der akademischen Bildungsmisere?: Das Niedersächsische AssistentInnen-Programm: »FH DoktorandInnen - Neue Wege in die Promotion«. FH-Journal Nordostniedersachsen, Heft 6, S. 12-15.

Mühlum, Albert (1998). Sozialarbeiterinnen und Sozialarbeiter im Promotionsverfahren. Erfahrungen und Perspektiven. Internet http://www.deutsche-gesellschaft-fuer-sozialarbeit.de/mit 11.shtml.

Schmitt, Rudolf (2008). Promotionen in der Sozialen Arbeit nach FH-Abschluss: Eine Handreichung für Promotionsinteressierte und Promovierende. Internet http://www.hs-zigr.de/ schmitt/ promotionen/v2/promotion_gesamt.pdf.

Weinbach, Heike (Hg.) (2001) unter Mitarbeit von Susanne Gerull, Nele Kleyer-Zey, Dietke Sanders, Claudia Streblow: FachhochschulabsolventInnen promovieren! Ein Leitfaden für SozialarbeiterInnen und PflegewirtInnen. Internet http://www.asfh-berlin.de/ uploads/media/leitfaden_promo_01.pdf. 
"Und wer Sterne entdecken will, lerne Brillen schleifen."

Friedrich Hebbel, deutscher Schriftsteller (1813-1863)

"Ernst zu nehmende Forschung erkennt man daran, dass plötzlich zwei Probleme existieren, wo es vorher nur eines gegeben hat. " Thorstein Bunde Veblen, amerikanischer Soziologe und Ökonom (1857-1929)

"Das Forschen ist an sich schon beinah die Entdeckung. Man findet immer, wenn man innig genug sucht; auf jede dringlich gestellte Frage kommt schließlich die Antwort. Oft zu unserem Schmerz."

Klaus Mann, deutscher Schriftsteller (1906-1949)

"Es hat niemand das Recht, Ideen und Forschungsergebnisse mitzuteilen, deren vollen Vollzug er nicht verantworten möchte. " Reinhold Schneider, deutscher Schriftsteller (1903-1958)

"Du. Forscher im Laboratorium. Wenn sie dir morgen befehlen, du sollst einen neuen Tod erfinden gegen das alte Leben, dann gibt es nur eins: Sag Neint"

Wolfgang Borchert, deutscher Schriftsteller (1921-1947)

»Ich möchte einmal in meinem Leben, nur einmal, einen Forschungsbericht sehen, der nicht endet mit

"Weitere Forschung ist dringend notwendig،." Gerhard Kocher, Schweizer Gesundheitsökonom (geb. 1939)

„Wir haben die Ergebnisse dieser Untersuchung im Rahmen einer wissenschaftlichen Publikation festgehalten. Das ergab einen Berg von Zahlen. Ich sehe jedoch keinen Sinn darin, sehr geehrte Anwesende, Ihnen diese Zahlen zu referieren. Wir haben alles zusammengezählt, und das Resultat war 31.280.411."

Beat Gloor, Schweizer Schriftsteller (geb. 1959) 